\title{
'||||||||||||||||||||||||||||||||||||||||||||||||||||||||||||||||||.
}

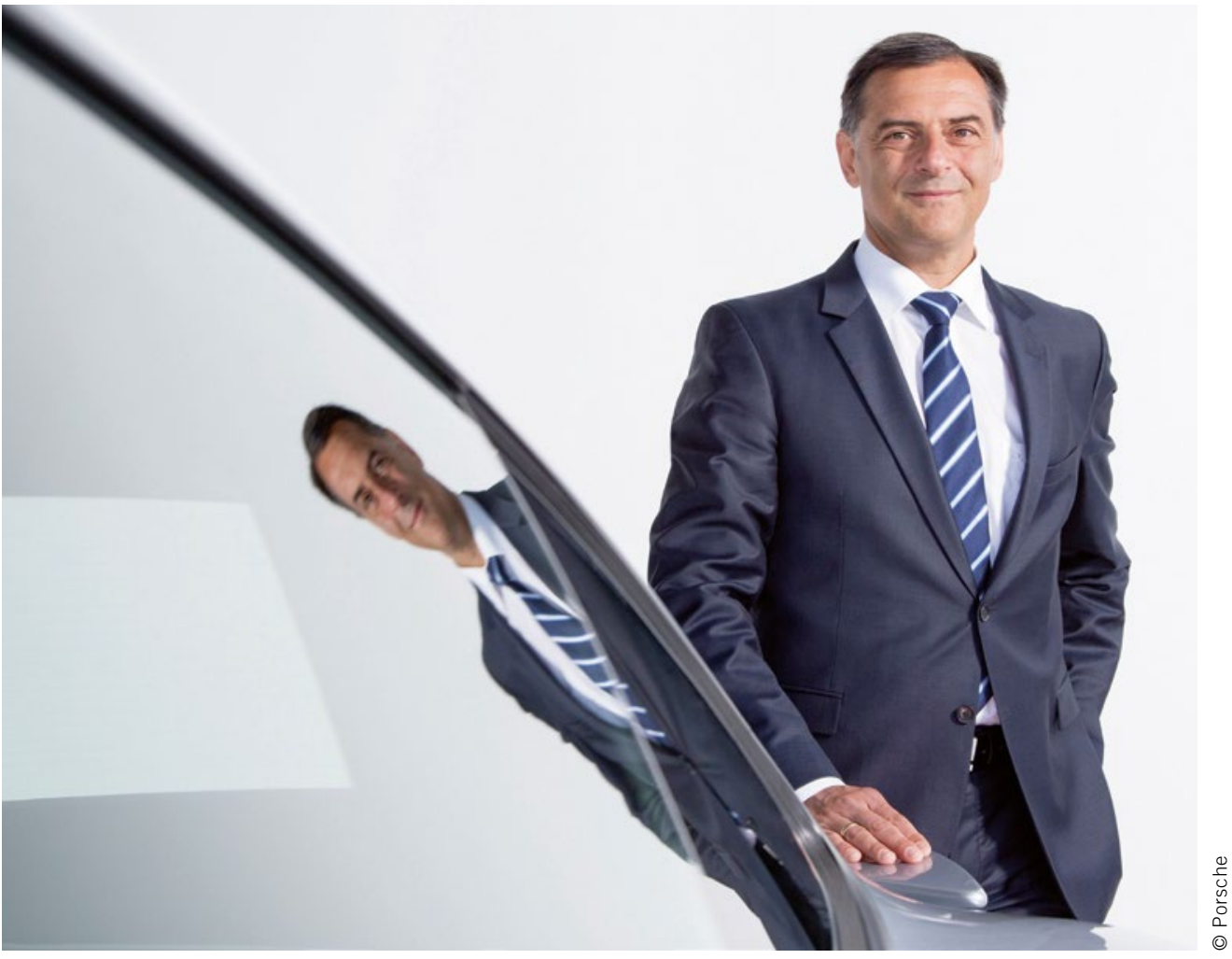

Dr.-Ing. Michael Steiner

Member of the Executive Board for Research and Development at Porsche AG, Member of the ATZ Advisory Board

\section{Charge Faster, Drive Longer}

Since the first electric vehicles and plug-in hybrids, the capacity of high-voltage batteries has been constantly improving. With the very next generation of vehicles, the E-models will be equal to their conventional competitors in terms of range. The increasing range of E-vehicles does however present a new dilemma - if the destination lies outside the action radius of the vehicle, then the battery must be recharged on the way, since an unchanged charging output means the charging time increases proportionally with the capacity of the battery. This means that drivers must take long breaks, hugely increasing the duration of a journey and quickly putting the advantages of a large range into perspective. In the future, charging speed will therefore be just as important as top speed.

To make the charging process as fast as conventional refuelling, significantly higher performance than that supplied by $50-\mathrm{kW}$ DC fast-charging stations is required. However, in systems with a voltage of $400 \mathrm{~V}$, the increased current means increased losses and large cable cross sections, which would make charging cumbersome and ineffective. For Porsche, the answer is the evolution of the $800-\mathrm{V}$ technology. Doubling the operating voltage to $800 \mathrm{~V}$ makes it possible to charge for a 400-km journey in $17 \mathrm{~min}$. It also makes cables easier to handle and reduces the weight of the vehicle.
The doubled operating voltage requires adjustments to many components of the electrical drivetrain. We can build on experience gained from the field of motorsport to make these adjustments, and draw on developments in industrial and railway technology for the power electronics and charging infrastructure. A new component, the HV booster, is required to ensure the compatibility of an $800-\mathrm{V}$ vehicle with existing $400-\mathrm{V}$ charging stations.

As is typical for Porsche, a performance-oriented design also applies to electric vehicles: This design must make it possible for a driver to charge for a high-speed journey in record time and continue their trip with no reduction in performance. This additional challenge can only be overcome with a systematic approach that combines connectivity, battery management and thermal management.

The Mission E concept with its 800-V technology represents our vision for future mobility: technological progress in the electrification of the drivetrain combined with digitalisation and connectivity, such as the ability to pay at the charging station. In the future, simply overcoming technical limitations will not be enough - the introduction of e-mobility is an economic task, as it will only be possible to provide real added value to the mobility users of tomorrow with a standardised infrastructure with a high level of availability. 